\section{COMPORTAMENTO ALIMENTAR E PERCEPÇÃO DA IMAGEM CORPORAL DE GESTANTES ATENDIDAS EM UM AMBULATÓRIO DE ALTO RISCO}

\author{
Eating behavior and body image perception of pregnant \\ women attending a high-risk outpatient center
}
Conducta alimentaria y percepción de la imagen corporal de embarazadas asistidas en un ambulatorio de alto riesgo

Artigo Original

\section{RESUMO}

Objetivo: Investigar o comportamento alimentar e a percepção da imagem corporal em gestantes atendidas em um ambulatório de alto risco. Métodos: Estudo quantitativo, observacional e transversal que incluiu 28 gestantes acima do peso atendidas na primeira consulta no ambulatório de nutrição em uma maternidade de Fortaleza-CE, nos meses de dezembro de 2010 a fevereiro de 2011. Utilizou-se um formulário pré-estabelecido, contendo dados de caracterização da amostra (socioeconômicos, obstétricos e nutricionais) e os questionários Binge Eating Scale (BES) para avaliar a compulsão alimentar e o Body Shape Questionnaire (BSQ) para a gravidade ou ausência de distúrbio de imagem corporal. As variáveis foram apresentadas como média \pm desvio padrão e frequência simples e percentual. Através da correlação de Pearson verificou-se a relação entre a imagem corporal e a compulsão alimentar, considerando-se $p<0,05$. Resultados: As gestantes analisadas apresentaram uma média de 29,4 $\pm 6,3$ anos e idade gestacional média de 24,6 $\pm 8,2$ semanas. Encontrou-se uma prevalência de $71,5 \%(n=20)$ de distúrbio de imagem corporal e de $17,8 \%$ $(n=5)$ de compulsão alimentar. Observou-se também uma correlação direta e significativa entre a percepção da imagem corporal e o grau de compulsão alimentar $(r=0,4358 ; p=0,020)$. Conclusão: A alta taxa de distúrbio da imagem corporal relacionada positivamente com uma considerável compulsão alimentar indica uma adaptação não favorável deste grupo de gestantes às alterações de peso, forma e tamanho corporal, próprias da gestação, configurando um grupo com necessidade de atenção especial por parte da equipe de profissionais.

Descritores: Gestantes; Imagem corporal; Transtorno da Compulsão Alimentar.

\section{ABSTRACT}

Objective: To investigate the eating behavior and body image perception in pregnant women attending a high-risk outpatient center. Methods: A quantitative, cross-sectional, observational study conducted with 28 overweight pregnant women attending the first consultation in the nutrition outpatient center of a maternity hospital in Fortaleza-CE, from December 2010 to February 2011. It has been used a pre-established form containing data on the characterization of the sample (socioeconomic, obstetric, and nutritional), the BES (Binge Eating Scale) to assess binge eating and BSQ (Body Shape Questionnaire) to assess the severity or absence of body image disorder. The variables were presented as mean \pm standard deviation and simple frequency and percentage. The Pearson's correlation was used to verify the relation between body image and binge eating, considering $p<0.05$. Results: The pregnant women studied had a mean age of $29.4 \pm 6.3$ years and mean gestational age of $24.6 \pm 8.2$ weeks. It was found a prevalence of $71.5 \%(n=20)$ of body image disorder and $17.8 \%(n=5)$ of binge eating. It was also observed a direct and significant correlation between the body image perception and the degree of binge eating $(r=0.4358, p=0.020)$. Conclusion: The high rate of body image disorder positively related to a significant binge eating indicates an unfavorable adjustment of this group of pregnant women to alterations in weight and body shape and size, which are inherent to pregnancy, standing out as group that needs special attention by the professional team.

Descriptors: Pregnant Women; Body Image; Binge-Eating Disorder.

\author{
Raquel Guimarães Nobre( ${ }^{(1)}$ \\ Ana Vaneska Passos Meireles ${ }^{(1)}$ \\ Julyanne Torres Frota ${ }^{(1)}$ \\ Raphael Marques de Miranda \\ $\operatorname{Costa}^{(2)}$ \\ Vanessa Fernandes Coutinho ${ }^{(3)}$ \\ Maria Miriam da Cunha Melo \\ Garcia $^{(1)}$ \\ Luciana Catunda Brito ${ }^{(4)}$
}

1) Maternidade Escola Assis Chateubriand MEAC - Fortaleza (CE) - Brasil

2) Hospital Geral Dr. Waldemar Alcântara HGWA - Fortaleza (CE) - Brasil

3) Universidade Gama Filho - UGF - Rio de Janeiro (RJ) - Brasil

4) Universidade Federal do Ceará - UFC Fortaleza (CE) - Brasil

Recebido em: 08/03/2013 Revisado em: 18/02/2014 Aceito em: 18/03/2014 


\section{RESUMEN}

Objetivo: Investigar la conducta alimentaria y la percepción de la imagen corporal de embarazadas asistidas en un ambulatorio de alto riesgo. Métodos: Estudio cuantitativo, observacional y transversal con 28 embarazadas con sobrepeso asistidas en la primera consulta del ambulatorio de nutrición de una maternidad de Fortaleza-Ce entre los meses de diciembre de 2010 y febrero de 2011. Se utilizó un formulario pre-establecido con datos para la caracterización de la muestra (socioeconómicos, obstétricos y nutricionales) y los cuestionarios BES (Binge Eating Scale) para evaluar la compulsión alimentaria y el BSQ (Body Shape Questionnaire) para evaluar la gravedad o ausencia del disturbio de la imagen corporal. Las variables fueron presentadas como media \pm desviación típica, frecuencia simple y porcentaje. A través de la correlación de Pearson se verificó la relación de la imagen corporal y la compulsión alimentaria considerando el $p<0,05$. Resultados: Las embarazadas evaluadas presentaron una media

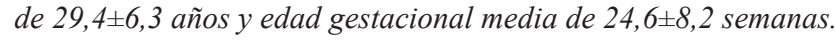
Se encontró una prevalencia del 71,5\% (n=20) de disturbio de imagen corporal y del 17,8\% $(n=5)$ de compulsión alimentaria. Se observó también una correlación directa y significativa de la percepción de la imagen corporal y el grado de compulsión alimentaria $(r=0,4358 ; p=0,020)$. Conclusión: La tasa elevada de disturbio de la imagen corporal relacionada positivamente con una considerable compulsión alimentaria indica una adaptación no favorable de este grupo de embarazadas a los cambios de peso, forma y tamaño corporal propias del embarazo lo que caracteriza un grupo con necesidad de atención especial de parte del equipo de profesionales.

Descriptores: Mujeres embarazadas; Imagen corporal: Trastorno por Atracón.

\section{INTRODUÇÃO}

A imagem corporal consiste na concepção interna e subjetiva sobre o corpo, que não se refere apenas à aparência física, mas envolve outros campos como a autopercepção corporal, as atitudes, as crenças e os sentimentos ${ }^{(1,2)}$.

O peso corporal é o aspecto central da imagem corporal nas culturas ocidentais, onde as mulheres sofrem considerável pressão social para serem magras, provocando, muitas vezes, uma insatisfação constante com a aparência física $^{(1,3)}$. Este quadro de insatisfação pode ser característico de distorção da imagem corporal, quando der origem a preocupações irracionais sobre defeitos em alguma parte do corpo, indicando alteração da percepção corporal do indivíduo ${ }^{(4)}$.

A distorção da imagem corporal compõe a etiologia dos transtornos alimentares (TA), que, por sua vez, se caracterizam por profundas alterações de padrão e comportamento alimentar, podendo se desenvolver como anorexia nervosa, bulimia nervosa, ou transtorno da compulsão alimentar periódica (TCAP) ${ }^{(1,5)}$.

Considerado pela OMS um problema de saúde pública $^{(6)}$, o TCAP consiste em um transtorno alimentar que se caracteriza por episódios de ingestão de grande quantidade de alimentos em um determinado período de tempo (até duas horas), seguido do sentimento de perda do controle sobre a vontade de comer. Para caracterizar o TCAP, esses episódios devem estar ocorrendo por pelo menos dois dias por semana nos últimos seis meses, e não serem associados a comportamentos compensatórios para evitar o ganho de peso, como ocorre na bulimia nervosa ${ }^{(7)}$.

Os transtornos alimentares podem ser considerados relativamente raros dentre a população geral (prevalência de 2,51\%), provavelmente porque há tendência de negar ou esconder este transtorno e ainda evitar ajuda profissional, contribuindo para uma possível subestimação dos dados dos estudos epidemiológicos nesse campo ${ }^{(8,9)}$.

Pesquisa envolvendo dados da Organização Mundial da Saúde, realizada com 24.124 participantes de 14 países, incluindo o Brasil, encontrou uma prevalência de TCAP de $1,9 \%$, e de $4,7 \%$ no Brasil (São Paulo), principalmente no sexo feminino(6). No Nordeste do Brasil, dados da Bahia mostram uma prevalência de TCAP em $5 \%$ na população geral, sendo maior no sexo feminino $(5,2 \%)$ e nos indivíduos que se autorreferiram obesos $(13,3 \%)^{(10)}$. No público das gestantes, a prevalência encontrada de TCAP é de $8,8 \%{ }^{(11)}$.

A gestação e o puerpério são períodos da vida da mulher que requerem atenção especial, pois envolvem, em um período relativamente curto (cerca de 40 semanas), inúmeras alterações físicas, hormonais, psíquicas e sociais $^{(2,12)}$. As mudanças no peso, forma e no tamanho corporal próprias da gestação podem gerar sentimentos negativos acerca da percepção corporal, influenciando o comportamento alimentar de tal forma, que os achados de TA em gestantes têm sido considerados cada vez mais comuns $^{(2,11,13,14)}$.

Em função da existência de poucos estudos envolvendo compulsão alimentar e percepção da imagem corporal em gestantes, objetivou-se investigar o comportamento alimentar e a percepção da imagem corporal em gestantes atendidas em um ambulatório de alto risco.

\section{MÉTODOS}

Trata-se de estudo quantitativo, observacional, transversal e analítico realizado ambulatório de atenção nutricional à gestante de alto risco, na Maternidade Escola Assis Chateaubriand, instituição tida como referência no estado do Ceará-Brasil em ginecologia e obstetrícia. 
Selecionou-se, por conveniência de modo consecutivo e não probabilístico, 28 gestantes no período de dezembro de 2010 a fevereiro de 2011. A amostra foi composta por mulheres obesas e com sobrepeso atendidas em primeira consulta no ambulatório de alto risco. O universo atendido no período de coleta foi de 44 pacientes, sendo excluídas $(n=16)$ as gestantes eutróficas ou desnutridas, e ainda aquelas com diagnóstico clínico de transtorno psiquiátrico.

Coletaram-se, com a ajuda de um formulário estruturado para este fim, informações envolvendo dados socioeconômicos (idade, estado civil, procedência, escolaridade e ocupação), obstétricos (patologias na gestação e idade gestacional) e nutricionais (Índice de Massa Corporal - IMC - pré-gravídico e gestacional no momento da consulta). Os dados nutricionais coletados foram analisados e classificados de acordo com os parâmetros recomendados pelo Ministério da Saúde para a avaliação do estado nutricional de gestantes ${ }^{(15)}$.

Para avaliação da imagem corporal e compulsão alimentar utilizaram-se os questionários validados e autoaplicáveis Body Shape Questionnaire (BSQ) ${ }^{(16)}$ e Binge Eating Scale (BES) ${ }^{(17)}$, sendo este último aplicado com a ajuda do pesquisador nutricionista.

A partir dos resultados do BSQ, que apresenta 34 itens avaliados por escores específicos para imagem corporal, as gestantes foram classificadas com: ausência de distúrbio de imagem $(\leq 80)$, distúrbio leve (81-110), moderado (111$140)$ e grave $(>140)^{(18)}$.

Analisou-se a compulsão alimentar nas gestantes pelo BES, que se caracteriza por uma ferramenta auto-aplicável e composta por escores distribuídos em 16 itens, os quais contemplam aspectos relacionados às manifestações comportamentais, sentimentos e cognições envolvidos em episódios de compulsão alimentar. A partir do somatório dos escores, classificou-se as participantes com compulsão alimentar: grave (escore $\geq 27$ ), moderada (escore entre $18 \mathrm{e}$ 26) e ausência de compulsão alimentar (escore $\leq 17)^{(18)}$.

As variáveis com escalas quantitativas foram apresentadas como média \pm desvio padrão e as variáveis com escala qualitativa, em frequência simples e percentual. Aplicou-se a correlação de Pearson para verificar a relação entre os resultados dos questionários BSQ e BES, com nível de significância de $5 \%(\mathrm{p}<0,05)$, utilizando o programa Primer of Biostatistics (versão 4.0).

O estudo foi aprovado pelo Comitê de Ética em Pesquisa da Maternidade Escola Assis Chateaubriand, sob o protocolo de $n^{\circ}$ 18/11, baseando-se na Resolução 196/96 da Comissão Nacional de Ética em Pesquisa (CONEP) do Conselho Nacional de Saúde (CNS).

\section{RESULTADOS}

Entre as gestantes avaliadas a idade média foi $29,4 \pm 6,3$ anos, com maior prevalência de mulheres casadas $(\mathrm{n}=11$; $39,3 \%)$, procedentes da capital $(n=28,100,0 \%)$, com ensino médio $(n=20,71,4 \%)$ e sem ocupação $(n=15,53,6 \%)$ (Tabela I).

Tabela I - Caracterização das gestantes atendidas em um ambulatório de alto-risco. Fortaleza-Ceará, 2011.

\begin{tabular}{lc}
\hline Variável & n (\%) \\
\hline Estado civil & \\
Casada & $11(39,3 \%)$ \\
$\quad$ Solteira & $6(21,4 \%)$ \\
$\quad$ Divorciada & $11(39,3 \%)$ \\
Procedência & \\
$\quad$ Capital & $28(100 \%)$ \\
Nível de Escolaridade & \\
$\quad$ Ensino fundamental & $6(21,4 \%)$ \\
Ensino médio & $20(71,4 \%)$ \\
Ensino superior & $2(7,1 \%)$ \\
Ocupação & $13(46,4 \%)$ \\
Sim & $15(53,6 \%)$ \\
Não & \\
\hline
\end{tabular}

Com relação aos dados obstétricos, a complicação mais frequente foi a pré-eclâmpsia $(\mathrm{n}=11,34,4 \%)$. A idade gestacional das participantes era de 24,6 $6 \pm 8,2$ semanas e $46,4 \%(n=13)$ estava no segundo trimestre da gravidez (Tabela II).

Tabela II - Dados obstétricos das gestantes atendidas em um ambulatório de alto-risco. Fortaleza-Ceará, 2011

\begin{tabular}{lc}
\hline Variável & n (\%) \\
\hline Complicações na gestação & $7(21,9 \%)$ \\
Nenhuma & $2(6,3 \%)$ \\
Gestação múltipla & $4(12,5 \%)$ \\
Hipertensão prévia & $11(34,4 \%)$ \\
Pré-eclâmpsia & $5(15,6 \%)$ \\
Diabetes & $1(3,1 \%)$ \\
Infecção urinária & $2(6,3 \%)$ \\
Outras* & \\
Idade gestacional & $3(10,7 \%)$ \\
$1^{\circ}$ trimestre & $13(46,4 \%)$ \\
$2^{\circ}$ trimestre & $12(42,9 \%)$ \\
$3^{\circ}$ trimestre & \\
\hline
\end{tabular}

*Outras: Anemia, placenta prévia.

De acordo com a avaliação nutricional pré-gravídica, o IMC médio das gestantes foi $34,8 \pm 9,1 \mathrm{~kg} / \mathrm{m}^{2}$, sendo que $85,8 \%(n=24)$ eram obesas e $42,9 \%(n=12)$ apresentando obesidade grau I. No momento da consulta esse panorama se manteve, quando as participantes estavam com IMC médio de $38,1 \pm 5,7 \mathrm{~kg} / \mathrm{m}^{2}$, com maior prevalência de gestantes obesas $(n=24,85,7 \%)$ (Tabela III). 
Tabela III - Perfil nutricional das gestantes atendidas em um ambulatório de alto-risco. Fortaleza-Ceará, 2011

\begin{tabular}{lc}
\hline Variável & n (\%) \\
\hline IMC* pré-gravídico\# & $3(11,1 \%)$ \\
Sobrepeso & $12(44,4 \%)$ \\
Obesidade grau I & $4(14,8 \%)$ \\
Obesidade grau II & $8(29,6 \%)$ \\
Obesidade grau III & \\
IMC gestacional & $4(14,3 \%)$ \\
Sobrepeso & $24(85,7 \%)$ \\
Obesidade
\end{tabular}

*IMC - Índice de Massa Corporal. \#n=27, pois 1 paciente não apresentava dados de peso pré-gravídico.

O resultado da aplicação do BSQ mostrou escore médio de $92,6 \pm 27,5$, com mais da metade da amostra ( $\mathrm{n}=20$, $71,5 \%)$ apresentando algum grau de distúrbio de imagem corporal, sendo a maior prevalência encontrada para a classificação distúrbio leve ( $\mathrm{n}=14,50,0 \%)$ (Tabela IV).

A aplicação do questionário BES revelou um escore médio de 10,6 $\pm 6,6$ e um percentual considerável de 17,9\% $(n=5)$ das gestantes apresentando compulsão alimentar moderada (Tabela IV).

Tabela IV - Análise da imagem corporal pelo Body Shape Questionnaire (BSQ) e da compulsão alimentar pelo Binge Eating Scale (BES) em gestantes atendidas em um ambulatório de alto-risco. Fortaleza-Ceará, 2011

\begin{tabular}{lc}
\hline Variável & $\mathrm{n}(\%)$ \\
\hline BSQ (Body Shape Questionnaire) & \\
Ausência de distúrbio de imagem & $8(28,6 \%)$ \\
Distúrbio leve & $14(50,0 \%)$ \\
Distúrbio moderado & $5(17,9 \%)$ \\
Distúrbio grave & $1(3,6 \%)$ \\
BES (Binge Eating Scale) & \\
Ausência de compulsão alimentar & $23(82,1 \%)$ \\
Compulsão alimentar moderada & $5(17,8 \%)$ \\
\hline
\end{tabular}

A insatisfação corporal esteve associada de forma direta e significativa com o grau de compulsão alimentar $(\mathrm{r}=0,4358 ; \mathrm{p}=0,020)$.

\section{DISCUSSÃO}

A gestação é um período delicado de mudanças corporais, principalmente ponderais, que tanto pode gerar um quadro de insatisfação corporal, evoluindo para um transtorno alimentar, quanto pode contribuir para uma melhora na satisfação corporal, existindo divergências na literatura sobre esta questão ${ }^{(19,20)}$.

Os resultados encontrados na amostra estudada sugerem que as gestantes de risco acima do peso apresentam insatisfação com a sua imagem corporal, ao se observar que mais da metade $(71,5 \%)$ apresentava algum grau de distúrbio de imagem corporal, principalmente em grau leve. Este resultado corrobora com estudo anterior que encontrou também um maior percentual de mulheres grávidas com distorção leve de imagem $(90 \%$ e $70 \%$, respectivamente em mulheres ativas e irregularmente ativas) ${ }^{(12)}$. Além disto, verificou-se uma relação inversa proporcional entre imagem corporal e autoestima $(\mathrm{r}=-0,61)$, indicando que quanto maior o escore do BSQ, menor a autoestima da gestante ${ }^{(12)}$. A partir deste achado, pode-se sugerir que o escore médio elevado encontrado no presente estudo $(92,6 \pm 27,5)$ possa ser indicativo de baixa autoestima destas gestantes.

Uma pesquisa realizada com mulheres grávidas apresentando diversos diagnósticos nutricionais encontrou que as gestantes acima do peso apresentavam um escore médio do BSQ maior $(74,94 \pm 23,36)$ do que aquelas que se apresentavam eutróficas $(62,95 \pm 22,8, \mathrm{p}<0.05)$, indicando maior prevalência de preocupações negativas com a imagem corporal em mulheres acima do peso ${ }^{(3)}$. Este achado pode justificar o maior escore encontrado na amostra estudada, composta exclusivamente de gestantes obesas e com sobrepeso.

Nesse contexto, tem-se mostrado que o IMC materno configura um preditor de autoestima e do comportamento alimentar, estando associado à insatisfação com a imagem corporal e à prática de dietas restritas ${ }^{(21,22)}$. Um destes estudos, que investigou gestantes com excesso de peso, encontrou que $70 \%$ destas mulheres não se identificavam como tendo um IMC acima do recomendado e que, aquelas que relataram insatisfação com a forma e o tamanho corporal eram mais suscetíveis a um ganho de peso excessivo durante a gestação(22). Assim, observa-se a importância do atendimento precoce a esse grupo, sendo o acompanhamento do IMC, ganho de peso e comportamento alimentar, pontos chaves do cuidado nutricional. $\mathrm{O}$ achado de que mulheres acima do peso apresentam maior chance de mudanças negativas na imagem corporal indica ser este um grupo no qual um tratamento direcionado possa ser ainda mais efetivo.

Especula-se que uma maior preocupação com a imagem corporal em gestantes também possa ser decorrente de diversos outros fatores como sintomas depressivos, tendência a comparar um corpo com outro, pressão sociocultural para ser magra e provocações dos outros ${ }^{(2)}$. Diante disso, ressalta-se a necessidade do conhecimento de todo o contexto onde a mulher encontra-se inserida, de modo a orientar a conduta dos profissionais, que devem trabalhar em equipe multidisciplinar para que o tratamento seja mais efetivo.

Com relação ao início do acompanhamento nutricional durante o pré-natal, os percentuais elevados encontrados 
para o início no segundo e terceiro trimestre gestacional $(\mathrm{n}=13,46,4 \%$ e $\mathrm{n}=12,42,9 \%$, respectivamente) geraram preocupação. Tem-se mostrado que as mulheres estão mais suscetíveis a elevados níveis de insatisfação corporal do início da gestação à metade do segundo trimestre, e que, assim ocorrendo, apresentam maiores chances de manterem esse quadro no terceiro trimestre, em associação com sintomas depressivos ${ }^{(2,23)}$. Isto reforça a importância do acompanhamento das gestantes desde cedo para detectar e tratar possíveis complicações de forma precoce e promover uma imagem corporal saudável e positiva nesse grupo $^{(23)}$.

A maternidade precipita várias problemáticas, principalmente aquelas relacionadas com o comportamento alimentar, podendo gerar um sentimento de perda de controle das quantidades ingeridas e mesmo do peso ${ }^{(24)}$.

Não existe um consenso na literatura sobre se a gestação contribui para o surgimento ou piora ${ }^{(24-26)}$ dos sintomas de TA, ou mesmo se ela teria um efeito redutor ou remissivo ${ }^{(27-29)}$ sobre os sintomas já presentes no período pré-gestacional ${ }^{(30)}$. Em estudo que envolveu uma amostra de 72.435 mulheres, observou-se que o início dos sintomas de TCAP pode ocorrer durante a gestação $(1,7 \%)$ ou iniciar antes e permanecer no curso da gravidez $(2,1 \%)$, ou ainda apresentarem redução na intensidade $(1,3 \%)^{(29)}$. A continuidade dos sintomas durante a gestação tem sido associada à preocupação excessiva com o peso antes e durante a gravidez ${ }^{(14)}$, e mulheres acima do peso apresentam maior tendência de remissão durante a gestação de um quadro prévio de $\operatorname{TCAP}^{(29)}$. Observa-se a versatilidade dos modos de incidência desse distúrbio em gestantes.

$\mathrm{Na}$ amostra pesquisada, encontrou-se um maior percentual para ausência de compulsão alimentar $(82,1 \%)$. Acredita-se que este achado possa ter sofrido influência da baixa escolaridade apresentada por algumas pacientes, tendo em vista a linguagem complexa deste questionário, mesmo com a ajuda do pesquisador. De acordo com os autores que validaram e traduziram o questionário (BES) para a língua portuguesa, os pacientes com baixo nível de escolaridade apresentam dificuldade em responder o questionário por acharem extenso e/ou muito complexo, apresentando problemas na leitura e no entendimento de algumas questões ${ }^{(17)}$.

A prevalência para compulsão alimentar moderada encontrada nesta pesquisa foi de $17,9 \%(\mathrm{n}=5)$. Tendo em vista que se sabe da associação entre TCAP e obesidade, sexo feminino e insatisfação corporal ${ }^{(10,14)}$, poder-se-ia esperar um percentual provavelmente maior de compulsão alimentar considerando-se o perfil da amostra estudada, formada por mulheres acima do peso, principalmente obesas e insatisfeitas, em sua maioria, com sua imagem corporal.

Entretanto, considerou-se este percentual significativo e digno de atenção pela equipe de saúde, principalmente pelas possíveis complicações obstétricas que podem ser resultantes de um quadro de TCAP, como hipertensão materna, maior duração do trabalho de parto e macrossomia, colocando a mãe e o feto em risco ${ }^{(31)}$.

Desse modo, a gestante com insatisfação corporal e com transtorno alimentar necessita de um tratamento multidisciplinar, principalmente no pré-natal, com foco nos hábitos alimentares e preocupação com peso e forma corporais $^{(27)}$. Assim, a investigação e diagnóstico precoce para TA durante a gestação são importantes para que sejam estabelecidas intervenções mais efetivas de modo a reduzir comportamentos que puderem ser prejudiciais à mãe e ao feto $^{(5,10,28)}$.

Pode-se considerar como limitações do estudo, o tamanho reduzido da amostra, decorrente do curto período de coleta, e a baixa escolaridade apresentada por algumas pacientes, além da complexidade da escrita e do tamanho extenso do questionário BES. Sugere-se a realização estudos subsequentes com ampliação da amostra e triagem para outros tipos de transtornos alimentares (bulimia e anorexia nervosa).

\section{CONCLUSÃO}

A alta taxa de distúrbio da imagem corporal relacionada positivamente com uma considerável compulsão alimentar indica uma adaptação não favorável deste grupo de gestantes às alterações de peso, forma e tamanho corporal, próprias da gestação, configurando um grupo com necessidade de atenção especial por parte da equipe de profissionais.

\section{REFERÊNCIAS}

1. Stenzel LM. A influência da imagem corporal no desenvolvimento e na manutenção dos transtornos alimentares. In: Nunes MA, Appolinario JC, Galvão AL, Coutinho W. Transtornos alimentares e obesidade. Porto Alegre: Artmed; 2006. p. 73-81.

2. Skouteris H, Carr R, Wertheim EH, Paxton SJ, Duncombe D. A prospective study of factors that lead to body dissatisfaction during pregnancy. Body Image. 2005;2(4):347-61.

3. Fox P, Ymaguchi C. Body image change in pregnancy: a comparison of normal weight and overweight primigravidas. Birth. 1997;24(1):35-40.

4. Schomer EZ, Kachani AT. Imagem Corporal. In: Cordás TA, Kachani AT. Nutrição em psiquiatria. Porto Alegre: Artmed; 2010. p. 107-18.

5. Dunker KLL; Alvarenga MS; Alves VPO. Transtornos alimentares e gestação - Uma revisão. J Bras Psiquiatr. 2009; 58(1):60-8. 
6. Kessler RC, Berglund PA, Chiu WT, Deitz AC, Hudson JI, Shahly V, et al. The prevalence and correlates of binge eating disorder in the World Health Organization World Mental Health Surveys. Biol Psychiatry. 2013;73(9):904-14.

7. Pisciolaro F, Azevedo AP. Transtorno da compulsão alimentar periódica. In: Cordás TA, Kachani AT. Nutrição em psiquiatria. Porto Alegre: Artmed; 2010. p. 167-80.

8. Smink FRE, Hoeken D, Hoek HW. Epidemiology of eating disorders: incidence, prevalence and mortality rates. Curr Psychiatry Rep. 2012;14(4):406-14.

9. Preti A, Girolamo G, Vilagut G, Alonso J, Graaf R, Bruffaerts R, et al. The epidemiology of eating disorders in six european countries: results of the ESEMeDWMH project. J Psychiatr Res. 2009;43(14):1125-32.

10. Mascarenhas MTL, Almeida MMG, Araújo TM, Prisco APK. Transtornos alimentares na população de 20 a 59 anos de Feira de Santana (BA), 2007. Cad Saúde Colet. 2011; 19(2):179-86.

11. Easter A, Bye A, Taborelli E, Corfield F, Schmidt U, Treasure J, et al. Recognising the symptoms: how common are eating disorders in pregnancy? Eur Eat Disord Rev. 2013;21(4):340-4.

12. Teixeira PC; Matsudo SMM; Almeida VS. Autoestima e imagem corporal de gestantes de acordo com o nível de atividade física. Rev Bras Ciênc Mov. 2008;16(1):547-655.

13. Meltzer-Brody S, Zerwas S, Leserman J, Holle AV, Regis T, Bulik C. Eating disorders and trauma history in women with perinatal depression. J Womens Health (Larchmt). 2011;20(6):863-70.

14 .Berg CK, Torgersen L, Holle AV, Hamer RM, Bulik CM, Reichborn-Kjennerud T. Factors associated with binge eating disorder in pregnancy. Int J Eat Disord. 2011;44(2):124-33.

15. Ministério da Saúde (BR), Secretaria de Atenção à Saúde, Departamento de Ações Programáticas Estratégicas. Gestação de alto risco: manual técnico. $5^{\text {a }}$ ed. Brasília: Ministério da Saúde, 2010.

16. Di Pietro M, Silveira DX. Internal validity, dimensionality and performance of the Body Shape Questionnaire in a group of Brazilian college students. Rev Bras Psiquiatr. 2008;31(1):21-4.

17. Freitas SR, Lopes CS, Coutinho W, Appolinario JC. Tradução e adaptação para o português da Escala de Compulsão Alimentar Periódica. Rev Bras Psquiatr. 2001;23(4):215-20.
18. Freitas SR. Instrumentos para a avaliação dos transtornos alimentares. In: Nunes MA, Appolinario JC, Galvão AL, Coutinho W. e colaboradores. Transtornos alimentares e obesidade. Porto Alegre: Artmed; 2006. p. 241-7.

19. Dunker KLL, Alvarenga M. Transtornos alimentares e gestação. In: Alvarenga M, Scagliusi FB, Philippi ST. Nutrição e transtornos alimentares: avaliação e tratamento. Barueri: Manole; 2010. p. 221-33.

20. Lotha KA, Bauera KW, Wallb M, Bergec J, NeumarkSztainera D. Body satisfaction during pregnancy. Body Image. 2011;8(3):297-300. Disponível em: http:// www.ncbi.nlm.nih.gov/pmc/articles/PMC3124621/

21. Shloim N, Hetherington M, Rudolf M, Feltbower R. Adjusting to motherhood: the importance of BMI in predicting maternal well-being, eating behaviour and feeding practice within a cross cultural setting. Appetite. No prelo 2014.

22. Sui Z, Turnbull D, Dodd J. Effect of body image on gestational weight gain in overweight and obese women. Women Birth. 2013;26(4):267-72.

23. Rauff EL, Downs DS. Mediating effects of body image satisfaction on exercise behavior, depressive symptoms, and gestational weight gain in pregnancy. Ann Behav Med. 2011;42(3):381-90.

24. Hubin-Gayte M, Squires C. Étude de l'impact de la grossesse sur les comportements alimentaires à travers l'utilisation du questionnaire SCOFF. Evol Psychiatr (Paris). 2012;77(2):201-12.

25. Cardoso JP, Pires AP. Perturbações do comportamento alimentar na gravidez: uma revisão. Psicol Reflex Crít. 2012:25(1):139-46.

26. Coker EL, Mitchell-Wong LA, Abraham SF. Is pregnancy a trigger for recovery from an eating disorder? Acta Obstet Gynecol Scand. 2013;92(12):1407-13.

27. Soares RM, Nunes MA, Schmidt MI, Giacomello A, Manzolli P, Camey $\mathrm{S}$ et al. Inappropriate eating behaviors during pregnancy: prevalence and associated factors among pregnant women attending primary care in southern Brazil. Int J Eat Disord. 2009;42(5):38793.

28. Harris AA. Practical advice for caring for women with eating disorders during the perinatal period. J Midwifery Womens Health. 2010;55(6):579-86.

29. Ulman TF, Holle AV, Torgersen L, Stoltenberg C, Reichborn-Kjennerud T, Bulik CM. Sleep disturbances and binge eating disorder symptoms during and after pregnancy. Sleep. 2012;35(10):1403-11. 
30. Santos AM, Piccolotto GB, Benute GRG, Santos NO, Lucia MCS, Francisco RPV. Transtorno alimentar e picacismo na gestação: revisão de literatura. Psicol Hosp. 2013;11(2):42-59.

31. Linna MS, Raevuori A, Haukka J, Suvisaari JM, Suokas JT, Gissler M. Pregnancy, obstetric, and perinatal health outcomes in eating disorders. Am J Obstet Gynecol. 2014;211(4):1-8.
Endereço para correspondência:

Raquel Guimarães Nobre

Rua Carlos Vasconcelos, 2956

Bairro: Aldeota

CEP: 60115-171 - Fortaleza - CE - Brasil

E-mail: raqgnobre@yahoo.com.br 